\title{
Versatile Roles of the Chromatin Remodeler CHD7 during Brain Development and Disease
}

\author{
Weijun Feng*, Chunxuan Shao and Hai-Kun Liu \\ Division of Molecular Neurogenetics, German Cancer Research Center, DKFZ-ZMBH Alliance, Heidelberg, Germany
}

CHD7 (Chromo-Helicase-DNA binding protein 7) protein is an ATP-dependent chromatin remodeler. Heterozygous mutation of the CHD7 gene causes a severe congenital disease known as CHARGE syndrome. Most CHARGE syndrome patients have brain structural anomalies, implicating an important role of $\mathrm{CHD7}$ during brain development. In this review, we summarize studies dissecting developmental functions of CHD7 in the brain and discuss pathogenic mechanisms behind neurodevelopmental defects caused by mutation of CHD7. As we discussed, CHD7 protein exhibits a remarkably specific and dynamic expression pattern in the brain. Studies in human and animal models have revealed that CHD7 is involved in multiple developmental lineages and processes in the brain. Mechanistically, CHD7 is essential for neural differentiation due to its transcriptional regulation in progenitor cells.

Keywords: CHD7, chromatin remodeler, CHARGE syndrome, brain development, mouse models

\section{OPEN ACCESS}

Edited by:

M. Albert Basson,

King's College London,

United Kingdom

Reviewed by:

Michael Telias,

University of California, Berkeley,

United States

Alessandro Sessa,

San Raffaele Hospital (IRCCS), Italy

${ }^{*}$ Correspondence:

Weijun Feng

w.feng@dkfz.de

Received: 31 July 2017 Accepted: 14 September 2017 Published: 28 September 2017

Citation:

Feng W, Shao C and Liu H-K (2017) Versatile Roles of the Chromatin Remodeler CHD7 during Brain Development and Disease.

Front. Mol. Neurosci. 10:309. doi: 10.3389/fnmol.2017.00309

\section{HETEROZYGOUS MUTATION OF CHD7 LEADS TO BRAIN DEVELOPMENTAL ANOMALIES}

The human CHD7 (Chromo-Helicase-DNA binding protein 7) is a long gene spanning approximately $189 \mathrm{~kb}$ at chromosome 8, containing 38 exons encoded for a large protein (2997 aa, about $336 \mathrm{kD}$ ). CHD7 protein is a member of CHD family of ATP-dependent chromatin remodelers. These enzymes utilize the energy from ATP hydrolysis to mobilize or relocate nucleosomes, thereby control DNA accessibility of chromatin. Chromatin remodeling is crucial for DNA-related biological processes such as transcription, chromosome segregation, DNA replication, and DNA repair (Clapier and Cairns, 2009). Not surprisingly, most chromatin remodelers are indispensable for normal development (Ho and Crabtree, 2010).

CHARGE syndrome (OMIM \#214800), initially described in 1979 (Hall, 1979; Hittner et al., 1979) and named in 1981 (Pagon et al., 1981), is a congenital disease with severe developmental defects in multiple organ systems. Two decades later, de novo mutations in the CHD7 gene were identified in CHARGE syndrome patients (Vissers et al., 2004), which turn out to be the major cause of this disease. Over $90 \%$ of patients with clinically typical CHARGE syndrome have heterozygous mutations in the CHD7 gene (Bergman et al., 2011). Moreover, mutations of CHD7 have also been identified in about 6\% of Kallmann syndrome (OMIM \#308700), a developmental disease characterized with IHH (idiopathic hypogonadotropic hypogonadism) and anosmia (Kim et al., 2008; Balasubramanian et al., 2014; Marcos et al., 2014). Up to now, 554 pathogenic CHD7 mutations have been identified in CHARGE syndrome patients. ${ }^{1}$ About $90 \%$ of these mutations

${ }^{1}$ www.chd7.org 
are nonsense, frame shift, and splice site mutations, which result in truncated $\mathrm{CHD} 7$ protein (Basson and van Ravenswaaij-Arts, 2015). In contrast, more than $70 \%$ of $C H D 7$ mutations in Kallmann syndrome are missense mutations (Balasubramanian et al., 2014; Marcos et al., 2014), which correlate with mild phenotypes in these patients as compared to CHARGE syndrome.

Several earlier review articles have comprehensive summarized the developmental roles of $\mathrm{CHD} 7$ in multiple organs affected in CHARGE syndrome patients (Layman et al., 2010; Zentner et al., 2010; Bergman et al., 2011). This review focuses on the function of CHD7 during brain development. Multiple structural defects in the brain of CHARGE syndrome patients have been reported, such as hypoplasia of olfactory bulb and cerebellum, agenesis of the corpus callosum, microcephaly and atrophy of the cerebral cortex (Lin et al., 1990; Tellier et al., 1998; Becker et al., 2001; Johansson et al., 2006; Sanlaville et al., 2006; Legendre et al., 2012; Yu et al., 2013; Hale et al., 2016). Among them, deficiency of olfactory bulb and sulci is the most frequent brain defect in CHARGE syndrome patients, as shown by Magnetic Resonance Imaging (MRI) (Chalouhi et al., 2005; Pinto et al., 2005; Blustajn et al., 2008). Because only small cohorts of patients were being examined in these studies and the phenotype of CHARGE syndrome is very heterogeneous, the overall percentage of patients having brain developmental anomalies is still not known. Nevertheless, brain structural defect has recently been proposed as minor criteria for the diagnosis of CHARGE syndrome (Hale et al., 2016). Consistent with defects in the brain, most CHARGE syndrome patients have certain degree of intellectual deficiency (Bergman et al., 2011). In Kallmann syndrome patients with CHD7 mutations, the common brain phenotype is hypoplasia of olfactory bulb and reduced number of GnRH (gonadotropin-releasing hormone) neurons in the hypothalamus (Marcos et al., 2014). Together, these observations clearly demonstrate that $\mathrm{CHD} 7$ is haploinsufficient for brain development.

\section{THE EXPRESSION OF CHD7 IN THE BRAIN IS VERY SPECIFIC AND DYNAMIC}

Consistent with specific brain defects caused by $\mathrm{CHD7}$ mutations, the CHD7 gene exhibits a spatial- and temporalspecific expression pattern during brain development. Expression of $\mathrm{CHD} 7$ in the human brain has been observed throughout development (Sanlaville et al., 2006). More detailed analysis of the expression of Chd7 has been done in mouse brain. Using in situ hybridization analysis, one study demonstrates that Chd7 is expressed as early as E8.5 (Embryonic day 8.5) in mouse brain regions including forebrain and midbrain neural fold, neural tube, and neuroepithelial prominence (Jiang et al., 2012). At E12.5 and E14.5, Chd7 is highly expressed in frontal cortex, medial ganglionic eminence, ventricular zone of medulla, and external granule zone of cerebellum (Bosman et al., 2005). A similar expression pattern of Chd7 was observed in $C h d 7^{G t /+}$ (Gt: Gene-trap) embryo, where the expression of $\beta$-galactosidase reporter closely mimics endogenous Chd7 (Hurd et al., 2007). One common observation from these studies is that the expression level of Chd7 is higher in the proliferating ventricular zone compared to the differentiated areas of the neuroepithelium (Figure 1A). In adult mouse brain, Chd7 shows a very specific and dynamic expression pattern in the adult neurogenic region subgranular zone (SGZ) in hippocampal dentate gyrus (DG) (Figure 1B). With immunostaining analysis, CHD7 is found to be expressed upon the activation of adult neural stem cells (NSCs), and is upregulated in transit progenitor cells and neuroblasts. In contrast, the expression of Chd7 is completely switched off in granule neurons in DG (Feng et al., 2013; Jones et al., 2015). This dynamic expression pattern of Chd7 in DG was confirmed in a recent single-cell RNA sequencing study whereas transcriptomes of EdU pulse-labeled individual neuronal cells were analyzed (Habib et al., 2016). Consistent with in vivo data showing the upregulation of Chd7 upon activation of adult NSCs, Chd7 is downregulated in cultured NSCs upon BMP4-induced quiescence (Martynoga et al., 2013).

In cerebellum, immunostaining results show that $\mathrm{CHD} 7$ is highly expressed in cerebellar granule cells throughout development and persists in adult cerebellum, in contrast to almost no expression in Purkinje neurons and Bergmann glia cells (Feng et al., 2017). In contrast to granule neurons in DG, the expression level of Chd7 is even higher in differentiated cerebellar granule neurons compared to granule neuron progenitors (GNPs) (Feng et al., 2017) (Figure 1C). Besides neuronal cells in the brain, while Chd7 is barely expressed in astrocytes, Chd7 is broadly expressed in oligodendrocyte (OL) lineage (He et al., 2016). Similar to cerebellar granule cells, the expression level of Chd7 in differentiated oligodendrocytes is higher than oligodendrocyte precursor cells (OPCs) (Figure 1C). With such a specific and dynamic expression pattern in the brain, the expression of Chd7 is expected to be tightly regulated. Mechanism behind is currently not known, however.

\section{LOSS-OF-FUNCTION STUDY IN MOUSE MODEL REVEALS AN IMPORTANT ROLE OF CHD7 DURING BRAIN DEVELOPMENT}

While xenopus (Bajpai et al., 2010), drosophila (Melicharek et al., 2010), and zebrafish models (Patten et al., 2012) have been established, mouse model is currently the predominant animal model for functional study of CHD7 and CHARGE syndrome. As shown in CHARGE syndrome patients, data from mouse studies confirm that CHD7 is haploidinsufficient for brain development (Table 1). Mice carrying germline lossof-function mutation of Chd7 have been generated with both ethylnitrosourea (ENU)-induced mutations and the gene-trap approaches. While homozygous loss-of-function mutation of Chd7 is embryonic lethal at E10.5, heterozygous mutant mice are viable but show phenotypes closely mimicking CHARGE syndrome (Bosman et al., 2005; Hurd et al., 2007). Analysis of E10.5 Chd7 homozygous gene-trap mutant embryo shows a reduction of the thickness of neuroepithelium in telencephalon and midbrain (Hurd et al., 2007), indicating that $C h d 7$ is required 
A

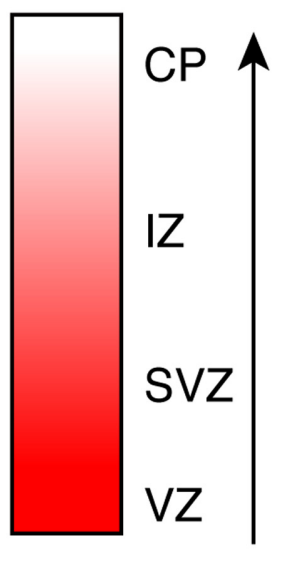

B
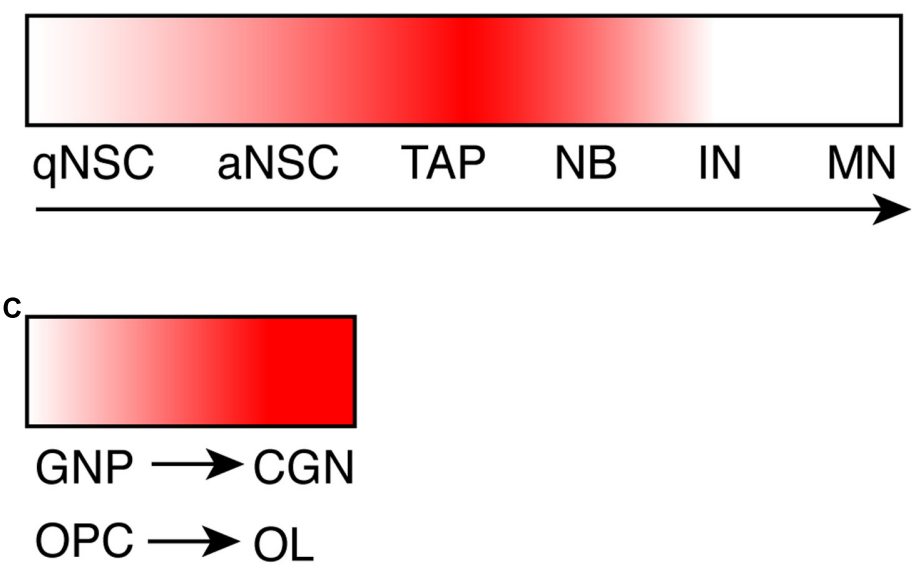

FIGURE 1 | The dynamic expression pattern of CHD7 during neurogenesis and oligodendrogenesis. (A) A cartoon shows the expression level of Chd7 transcript during embryonic neurogenesis, based on in situ hybridization data. (B) A cartoon shows the expression level of CHD7 protein in adult neurogenic lineage in hippocampal dentate gyrus, based on immunostaining results. (C) A cartoon shows the increase of CHD7 expression upon differentiation of GNP and OPC, based on immunostaining results. The degree of redness reflects the expression level of Chd7. Arrows show the direction of differentiation. VZ, ventricular zone; SVZ, subventricular zone; IZ, intermediate zone; CP, cortical plate; qNSC, quiescent neural stem cell; aNSC, active NSC; TAP, transit amplifying progenitor; NB, neuroblast; IN, immature neuron; MN, mature neuron; GNP, granule neuron progenitor; CGN, cerebellar granule neuron; OPC, oligodendrocyte precursor cell; OL, oligodendrocyte.

TABLE 1 | Brain phenotype in Chd7 loss-of-function mutant mouse models.

\begin{tabular}{|c|c|c|c|}
\hline Mouse lines & Mouse brain phenotype & Relevant CHARGE phenotype & Reference \\
\hline $\begin{array}{l}\text { Chd } 7^{G t}(S 20-7 E 1) / G t(S 20-7 E 1) \\
\text { (Gene-trap reporter inserted in } \\
\text { exon 1) }\end{array}$ & $\begin{array}{l}\text { E10.5 lethal, hypoplasia of the } \\
\text { neuroepithelium }\end{array}$ & Cognitive disability & Hurd et al., 2007 \\
\hline Chd7 ${ }^{\mathrm{Gt}}(\mathrm{S} 20-7 E 1) /+$ & Small olfactory bulb (OB) & Hyposmia & Layman et al., 2009 \\
\hline $\begin{array}{l}\text { Chd7 Whi/+ (p.W973X) } \\
\text { Chd7Gt(S20-7E1)/+ }\end{array}$ & $\begin{array}{l}\text { OB hypoplasia; decreased GnRH } \\
\text { neurons in the hypothalamus }\end{array}$ & $\begin{array}{l}\text { Anosmia; Genital hypoplasia; Puberty } \\
\text { delay }\end{array}$ & $\begin{array}{l}\text { Bergman et al., 2010; Layman et al., } \\
2011\end{array}$ \\
\hline Chd7COA1/+ (p.K719X) & $\begin{array}{l}\text { Hypoplasia of OB; Telencephalic midline } \\
\text { defects; Reduced cerebral cortex }\end{array}$ & Hyposmia; Cognitive disability & Jiang et al., 2012 \\
\hline $\begin{array}{l}\text { Nestin-CreERT2::Chd7 }{ }^{f / f} \\
\text { Ubc-CreERT2::Chd7 }{ }^{f / f} \\
\text { Glast-CreERT2::Chd7 }{ }^{f / f}\end{array}$ & Defects of adult neurogenesis & Cognitive disability; Hyposmia & $\begin{array}{l}\text { Feng et al., 2013; Micucci et al., 2014; } \\
\text { Jones et al., } 2015\end{array}$ \\
\hline $\begin{array}{l}\text { Chd7Gt(XK403)/+ (Gene-trap } \\
\text { reporter inserted in intron 36) } \\
\text { Atoh1-Cre::Chd7f/f } \\
\text { Nestin-Cre::Chd } 7^{f / f}\end{array}$ & $\begin{array}{l}\text { Vermis hypoplasia; Purkinje cell } \\
\text { heterotopia }\end{array}$ & $\begin{array}{l}\text { Vermis hypoplasia; Purkinje cell } \\
\text { heterotopia }\end{array}$ & $\begin{array}{l}\text { Yu et al., 2013; Feng et al., 2017; } \\
\text { Whittaker et al., } 2017\end{array}$ \\
\hline $\begin{array}{l}\text { Olig1-Cre::Chd7 }{ }^{f / f} \\
\text { Pdgfr-CreERT2::Chd7 }{ }^{f / f}\end{array}$ & Defect of myelination & White matter defects & He et al., 2016 \\
\hline
\end{tabular}

for early brain development. Heterozygous Chd7 loss-of-function mutant mice show defects in different brain regions. The most frequent defect is the absence or hypoplasia of olfactory bulb (Layman et al., 2009; Bergman et al., 2010; Jiang et al., 2012). Other brain anomalies identified in these mice include reduced number of GnRH neurons in the hypothalamus (Layman et al., 2011), defects in the development of telencephalic midline and reduction of the thickness of cerebral cortex (Jiang et al., 2012).

Ablation of Chd7 in germline is embryonic lethal at E10.5, which prevents the study of its function in brain development during later stage. In order to further dissect the function of Chd7 in brain development, several labs have applied Chd7 conditional knockout (CKO) mouse lines (Chd7flox/flox $)$ (Table 1). Two
Chd $7^{\text {flox/flox }}$ mouse lines have been used, with either exon 2 or 3 being floxed (Hurd et al., 2010; Feng et al., 2013). Upon cre-mediated recombination, the $\mathrm{CHD} 7$ protein is ablated in CKO mouse. Compared to mice carrying germline lossof-function mutation of Chd7, key advantage of Chd7 CKO mouse line is that it allows researchers to dissect the function of Chd7 in specific cell lineage during brain development in a spatial- and temporal-specific manner. As discussed below, crucial functions of Chd7 in adult neurogenesis, cerebellar development, and CNS myelination have been revealed by the CKO approach.

Adult neurogenesis occurs in restricted mouse brain regions including SGZ of DG in hippocampus and subventricular 
zone (SVZ) of lateral ventricle (Gage, 2000). Given neurogenic mechanism in adult is similar to embryonic stage, adult neurogenesis provides an ideal system to study neurodevelopment. Moreover, while germline homozygous loss-of-function mutations of many neurodevelopmental genes are embryonic lethal, adult neurogenesis, in the other hand, allows the study of homozygous gene-silencing mutations because majority of them do not affect animal survival. Three independent studies demonstrated that loss of Chd7 in adult NSCs leads to a decline of adult neurogenesis (Feng et al., 2013; Micucci et al., 2014; Jones et al., 2015). In Chd7 CKO brain, the number of newborn neurons reduces, and mutant neurons show clear defects in dendritic development. Strikingly, voluntary running, a positive stimulus of adult neurogenesis in hippocampus, could rescue hippocampal neurogenic defects, including both the amount and the dendritic development of newborn neurons in Chd7 CKO mice (Feng et al., 2013). Mutation of Chd7 in adult NSCs was also shown to result in a loss of quiescent stem cells in DG, thereby an exhaustion of NSC pool (Jones et al., 2015). Dissecting mechanism behind the adult neurogenic defect in Chd7 CKO mice reveals the function of CHD7 in neurodevelopment, which may help us to understand the cognitive deficiency that is frequently observed in CHARGE syndrome patients.

Structural defects in cerebellum occur frequently in CHARGE syndrome patients (Becker et al., 2001; Sanlaville et al., 2006; Legendre et al., 2012; Yu et al., 2013). Recent mouse studies have provided valuable insights into mechanism behind the pathogenesis of cerebellar anomalies caused by loss-of-function of Chd7. Heterozygous gene-trap mutation of Chd7 leads to reduced expression of key signaling molecule Fgf8 (Fibroblast growth factor 8 ) in isthmus organizer ( $\mathrm{IsO}$ ), an organizer region that directs cerebellar development during early embryonic stage (between E8 and E9). Importantly, heterozygous loss-offunction mutations of both Chd7 and Fgf8 show a synergistic effect in cerebellar development, resulting in severe vermis aplasia (Yu et al., 2013). While this study provides evidence showing the important role of Chd7 during early embryonic development of cerebellum, recent studies have revealed that Chd7 is also required for cerebellar development at later stages. Using the Atoh1-Cre::Chd $7^{\text {flox/flox }}$ mouse line to knockout Chd7 specifically in GNPs, two independent studies clearly reveal that Chd7 mutant animals exhibit cerebellar hypoplasia and massive Purkinje cell heterotopia (Feng et al., 2017; Whittaker et al., 2017). Importantly, highly similar phenotypes were observed in CHARGE syndrome pre-fetuses and patients (Legendre et al., 2012; Yu et al., 2013). Ablation of Chd7 in Purkinje cell progenitors in the Ptfla-Cre::Chd $7^{\text {flox/flox }}$ mice does not result in any obvious cerebellar developmental defect (Feng et al., 2017), excluding an essential role of Chd7 in Purkinje cell lineage. Together, these findings strongly implicate that dysfunction of CHD7 in cerebellar granule cell lineage leads to cerebellar defects in CHARGE patients.

Consistent with the specific expression of Chd7 in OL lineage, CHD7 has been shown to play a crucial role in myelination during brain development and remyelination after drug-induced demyelination in adult mice (He et al., 2016). Further mechanistic study show that Chd7 is required for differentiation and maturation of OLs. The function of CHD7 in oligodendrogenesis may help to explain the structural defects in white matter and corpus callosum of CHARGE syndrome patients.

\section{LOSS OF CHD7 IN THE MOUSE BRAIN CAUSES COMMON CELLULAR PHENOTYPE}

As discussed above, CHD7 is involved in both neurogenesis and oligodendrogenesis, highlighting its versatile roles during brain development. On the other hand, loss of Chd7 in different cell lineages in mouse brain causes similar cellular phenotype. First, loss of Chd7 leads to defect in terminal differentiation of mouse neural progenitor cells. Ablation of Chd7 in mouse adult NSCs or cerebellar GNPs impairs terminal differentiation of granule neurons in DG and cerebellum, respectively (Feng et al., 2013, 2017). Similarly, ablation of Chd7 in mouse OPCs leads to defects in the differentiation of OLs (He et al., 2016). Second, Chd7 seems to be dispensable for both the generation and the proliferation of neural progenitor cells. Actually, ablation of Chd7 in adult NSCs in both Nestin-CreERT2::Chd $7^{\text {flox/flox }}$ and Glast-CreERT2::Chd flox/flox mice leads to a mild increase of cell proliferation in the SGZ (Feng et al., 2013; Jones et al., 2015). The specification and proliferation of cerebellar GNPs are not affected in Atoh1-Cre::Chd7flox/flox mice (Feng et al., 2017). Knockout of Chd7 in OL lineage in Olig1-Cre::Chd7flox/flox mice, does not affect the generation and proliferation of OPCs (He et al., 2016). Third, loss of Chd7 in neural progenitor cells leads to increased cell death. Increased cell death was observed upon loss of Chd7 in both adult neurogenic regions and cerebellum in mouse (Feng et al., 2013, 2017; Whittaker et al., 2017). Cultured Chd7 mutant mouse GNPs are more prone to cell death upon differentiation (Feng et al., 2017). Interestingly, loss of Chd7 was shown to activate p53-dependent induction of apoptosis during embryonic development, and inhibition of p53-dependent apoptosis could partially rescue developmental defects in Chd7 knockout embryo (Van Nostrand et al., 2014). Whether p53-dependent activation of cell death occurs during adult neurogenesis and cerebellar development need to be tested. In summary, CHD7 seems to have a similar cellular function in different cell lineages during brain development. CHD7 is essential for both neurogenesis and oligodendrogenesis via controlling the differentiation of neural progenitor cells. Loss of Chd7 in progenitor cells has mild effect on cell proliferation, but leads to the increase of cell death.

\section{KEY TARGET GENES OF CHD7 DURING BRAIN DEVELOPMENT}

Consistent with the known function of chromatin remodelers in transcriptional regulation, a common molecular function of CHD7 in neural cells is controlling gene expression. Multiple target genes of $\mathrm{CHD} 7$ during brain development have been 
TABLE 2 | Key target genes of CHD7 in mouse neural cells.

\begin{tabular}{|c|c|c|c|c|}
\hline Target genes & Neural cells & Direct binding of CHD7 & Rescue & Reference \\
\hline Jag1, Gli2, Gli3, MycN, Hes5 & Neural stem cells (NSCs) & Promoter and enhancer, ChIP-seq & n.d. & Engelen et al., 2011 \\
\hline Sox4, Sox11 & Adult NSCs & Promoter, ChIP-qPCR & Yes & Feng et al., 2013 \\
\hline Rarb, Rxrg, Neurod1 & Subventricular zone (SVZ) NSCs & Promoter, ChIP-qPCR & Yes & Micucci et al., 2014 \\
\hline Hes5 & Subgranular zone (SGZ) NSCs; NSCs & Promoter, ChIP-seq & Yes & Engelen et al., 2011; Jones et al., 2015 \\
\hline Osterix, Creb3/2 & Oligodendrocytes & Promoter and enhancer, ChIP-seq & Yes & He et al., 2016 \\
\hline Otx2, Gbx2 & Cells in rhombomere 1 & Enhancers, ChIP-qPCR & n.d. & Yu et al., 2013 \\
\hline Reelin & Cerebellar GNPs & Enhancers, ChIP-seq & Yes & Feng et al., 2017; Whittaker et al., 2017 \\
\hline
\end{tabular}

identified (Table 2). Using shRNA-mediated gene knock down approach in cultured mouse NSCs, Engelen et al. (2011) show that CHD7 functions as a cofactor of the SoxB family of transcription factor SOX2 to activate multiple developmental disease-relevant genes like Jag1, Gli2, Gli3, and $M y c N$. In adult mouse NSCs, CHD7 is required for the activation of two SoxC family of transcription factors SOX4 and SOX11, and overexpression of SOX4 and SOX11 could largely rescue the neuronal differentiation defect in Chd7 mutant NSCs (Feng et al., 2013). Another study show that CHD7 activates the expression RA (retinoic acid) signaling receptors Rarb and Rxrg, and RA treatment could partially rescue neuronal differentiation defect in neurosphere derived from the SVZ of Chd7 CKO mice (Micucci et al., 2014). Also, CHD7 was shown to activate Notch effector HES5 in quiescent adult mouse NSCs, which is known to be required for the maintenance of NSC quiescence (Jones et al., 2015). During early cerebellar development, CHD7 was found to activate and repress the expression of Gbx2 and Otx2, respectively, which results in the downregulation of $\mathrm{Fgf8}$ in IsO (Yu et al., 2013). During postnatal development of cerebellum, two independent studies reported reelin, an essential gene for neuron migration, as a key target gene of Chd7 (Feng et al., 2017; Whittaker et al., 2017). Using the Nestin-Reln mouse line, Whittaker et al. (2017) show that overexpression of reelin during brain development partially rescues defects of Chd7 mutant cerebellum. Using RNA-seq and ChIP-seq (Chromatin immunoprecipitation with high throughput sequencing) analysis in differentiating OLs, CHD7 was found to regulate genes like Osterx and Creb3l2, both of them are required for both OL differentiation and bone formation (He et al., 2016). Except the Otx2 gene, CHD7 functions as a transcriptional activators for its target genes. Genome-wide analysis including RNAseq and ChIP-seq in GNPs and OLs support the notion that $\mathrm{CHD} 7$ is required for the activation of gene expression during neural differentiation ( $\mathrm{He}$ et al., 2016; Feng et al., 2017).

\section{THE MOLECULAR FUNCTION OF CHD7 IN NEURAL CELLS}

Data from several CHD7 ChIP-seq studies have shown that CHD7 protein preferentially binds to distal regulatory elements in different cells including ESCs (embryonic stem cells), NPCs, and GNPs (Schnetz et al., 2009, 2010; Ram et al., 2011; Feng et al., 2017). In particular, two studies have shown the association of CHD7 to super-enhancers in human ESCs and mouse GNPs (Hnisz et al., 2013; Feng et al., 2017). Given super-enhancers are associated with genes establishing cell identity, these findings implicate important role of $\mathrm{CHD} 7$ in cell fate determination. As an example, two super-enhancers are present at the Chd7 gene in mouse GNPs (Feng et al., 2017), consistent with the important role of $\mathrm{CHD} 7$ in these cells. Using ATAC-seq (transposaseAccessible Chromatin with high throughput sequencing), Feng et al. (2017) show loss of Chd7 leads to specific alteration of open chromatin structure in distal regulatory elements of Chd7 target genes. How CHD7 is recruited to specific targets is still an open question. Given CHD7 protein does not have DNA binding specificity, it is believed that sequence-specific transcription factors recruit $\mathrm{CHD} 7$ to its target genes. Along with this line, several transcription factors have been reported to interact with CHD7. In mouse NSCs, knock down of Sox2 impairs the binding of CHD7 to its target, indicating that Sox 2 is involved in the recruitment of Chd7 (Engelen et al., 2011). In differentiating OLs, CHD7 is shown to interact with a SoxE family of transcription factor SOX10, and to colocalize with SOX10 genome-wide (He et al., 2016). The requirement of SOX10 for the targeting of CHD7 in OLs has not been shown in this study, however.

As expected, the chromatin remodeling activity is important for the function of CHD7 during development. Most of CHD7 mutations in CHARGE syndrome patients result in truncated $\mathrm{CHD} 7$ protein that apparently lost its ATPase and chromatin remodeling activities. Importantly, Bouazoune and Kingston (2012) observed that several patients-derived missense CHD7 mutations lead to reduction or loss of its ATPase and remodeling activity in vitro. Intriguingly, $\mathrm{CHD} 7$ interacts with other chromatin remodelers. Multiple evidences suggest a possible functional interaction between $\mathrm{CHD} 7$ and the BAF (Brg1/Brahma-associated factors) complex. First, in human neural crest cells, CHD7 is associated with multiple subunits of the PBAF (Polybromo- and Brg1/Brahma-associated factors) complex (Bajpai et al., 2010). CHD7 and PBAF colocalize to distal regulatory elements of key neural crest transcription factors, and synergistically activate neural crest gene expression (Bajpai et al., 2010). Consistently, in neural crest-derived melanocyte, Brg1-CHD7-containing PBAF complex interacts with and facilitates the function of the master transcription factor MITF (Microphthalmia-associated transcription factor) (Laurette et al., 2015). Second, in OLs, BRG1 was shown to activate the 
expression of Chd7 (He et al., 2016). Third, phenotypes of Brg1 CKO brain in adult neurogenesis and cerebellar development (Ninkovic et al., 2013; Moreno et al., 2014) are similar to Chd7 CKO brain. Moreover, yeast two-hybrid screening has identified CHD8, another CHD family chromatin remodeler, as an interacting partner of CHD7 (Batsukh et al., 2010). The interaction between CHD7 with BRG1 and CHD8 was confirmed in HEK293T cells using coimmunoprecipitation coupled to mass spectrometry (Feng et al., 2017). How chromatin remodelers function together is an open question. One stimulating study show that three chromatin remodelers BRG1, CHD4, and SNF2H colocalize to a substantial portion of sites on chromatin, and the DNA accessibility of many regions requires a combined activity of several remodelers (Morris et al., 2014). It is worthy to investigate the potential cooperative or counteractive functions of $\mathrm{CHD} 7$ with BRG1 and CHD8 during brain development.

Another interesting molecular mechanism revealed from a recent study is the cooperative function of $\mathrm{CHD} 7$ and DNA topoisomerase TOP2B in transcriptional regulation (Feng et al., 2017). Increasing evidences show the cooperative role of chromatin remodelers and DNA topoisomerases. In mouse ESCs, BAF complex was shown to regulate DNA decatenation during mitosis by recruiting DNA topoisomerase TOP2A (Dykhuizen et al., 2013). One recent study from the same lab demonstrates that TOP2B synergizes with the BAF complex to resolve facultative chromatin to accessible chromatin (Miller et al., 2017). Another recent study shows that BRG1 is required for the recruitment of Topoisomerase 1 in B-cell line (Husain et al., 2016). Importantly, enzymatic activities of TOP 1 and TOP $2 B$ are absolutely required for the transcription of long genes (>100 kb in gene length) in neurons (King et al., 2013). Many of expressed long genes in neurons are essential neuronal genes, with their dysfunction results in various human neurological disorders. Feng et al. (2017) demonstrate that CHD7 recruits TOP2B to facilitate the transcription of long genes in cerebellar granule neurons, including the reelin gene. Consistently, ablation of Top $2 b$ specifically in mouse forebrain results in a reelin-deficient phenotype in cerebral cortex (Lyu and Wang, 2003).

\section{FUTURE PERSPECTIVE}

One of remaining questions concerning the function of CHD7 in the brain is whether CHD7 is required for the function of mature neurons. As discussed before, the expression of $C h d 7$ is switched off in most types of mature neurons in the brain during neurogenesis. However, CHD7 is highly expressed in cerebellar granule neurons in adult mouse and human brain (Feng et al., 2017), and some interneurons in the olfactory bulb of adult mouse (Micucci et al., 2014). The selective expression of Chd7 indicates that it may be involved in the function of these mature neurons. Studying the function of CHD7 in adult brain may help us to understand neuronal behavior abnormality frequent observed in CHARGE syndrome patients.

Several key questions regarding molecular function of CHD7 in the cell remains to be answered. First, what is pathogenic mechanisms of CHD7 missense mutations? Study of missense mutations could provide important insight into the molecular and biochemical function of CHD7 protein. Recent development of structure analysis of chromatin remodelers may provide crucial mechanistic insight of missense mutations of CHD7. For instance, the chromo domain of CHD1 has been shown to be structurally required for the activity of its ATPase activity (Sundaramoorthy et al., 2017). Given the chromo domains in CHD family of chromatin remodelers are conserved, this finding may provide a mechanistic answer for the apparent loss of ATPase activity of one chromo domain mutation CHD7 S834F (Bouazoune and Kingston, 2012). Second, what is the exact function of CHD7 at enhancers? It is tempting to speculate that the nucleosome remodeling activity of $\mathrm{CHD} 7$ facilitates the transcription activity at enhancers. This hypothesis remains to be experimentally tested. Third, does CHD7 functions alone or within a complex? As examples, CHD1 functions as a monomer (Tran et al., 2000; Lusser et al., 2005), in contrast, CHD4 functions within the NuRD (Nucleosome remodeling Deacetylase) complex (Zhang et al., 1998). Solid biochemical assays need to be performed to answer this question.

It is worthy to notice that recent unprecedented development of technology in the field of human cell-based disease modeling. It has already become a routine practice to derive human induced pluripotent stem cells (iPSCs) from patients. The full reservoir of differentiation capacity enables iPSCs as an excellent cell model for disease modeling. Recent development of genome editing tools such as CRISPR-Cas technology has in principle enabled the genome editing at anywhere of the genome in any cell. In particular, genome editing in human iPSCs has enormous application in biomedical research (Hockemeyer and Jaenisch, 2016). Moreover, the recent development of 3D-based human brain organoid culture system has largely improved our ability to model human brain development in tissue culture dish (Lancaster et al., 2013). These state-of-art human cellbased technologies have been applied to model human brain development and neurological disorders. Study the function of $\mathrm{CHD} 7$ using this approach is expected to advance our understanding of role of $\mathrm{CHD} 7$ in brain development and disease.

\section{AUTHOR CONTRIBUTIONS}

All authors listed have made a substantial, direct and intellectual contribution to the work, and approved it for publication.

\section{FUNDING}

This work was supported by the Helmholtz Young Investigator Award (VH-NG-702, to $\mathrm{H}-\mathrm{KL}$ ), the Deutsche Forschungsgemeinschaft (LI 2140/1-1, to H-KL), and the DKFZ Intramural Grant Program (to WF). 


\section{REFERENCES}

Bajpai, R., Chen, D. A., Rada-Iglesias, A., Zhang, J., Xiong, Y., Helms, J., et al. (2010). CHD7 cooperates with PBAF to control multipotent neural crest formation. Nature 463, 958-962. doi: 10.1038/nature08733

Balasubramanian, R., Choi, J. H., Francescatto, L., Willer, J., Horton, E. R., Asimacopoulos, E. P., et al. (2014). Functionally compromised CHD7 alleles in patients with isolated GnRH deficiency. Proc. Natl. Acad. Sci. U.S.A. 111, 17953-17958. doi: 10.1073/pnas.1417438111

Basson, M. A., and van Ravenswaaij-Arts, C. (2015). Functional insights into chromatin remodelling from studies on CHARGE syndrome. Trends Genet. 31, 600-611. doi: 10.1016/j.tig.2015.05.009

Batsukh, T., Pieper, L., Koszucka, A. M., von Velsen, N., Hoyer-Fender, S., Elbracht, M., et al. (2010). CHD8 interacts with CHD7, a protein which is mutated in CHARGE syndrome. Hum. Mol. Genet. 19, 2858-2866. doi: 10.1093/ $\mathrm{hmg} / \mathrm{ddq} 189$

Becker, R., Stiemer, B., Neumann, L., and Entezami, M. (2001). Mild ventriculomegaly, mild cerebellar hypoplasia and dysplastic choroid plexus as early prenatal signs of CHARGE association. Fetal Diagn. Ther. 16, 280-283. doi: $10.1159 / 000053928$

Bergman, J. E., Bosman, E. A., van Ravenswaaij-Arts, C. M., and Steel, K. P. (2010). Study of smell and reproductive organs in a mouse model for CHARGE syndrome. Eur. J. Hum. Genet. 18, 171-177. doi: 10.1038/ejhg.2009.158

Bergman, J. E., Janssen, N., Hoefsloot, L. H., Jongmans, M. C., Hofstra, R. M., and van Ravenswaaij-Arts, C. M. (2011). CHD7 mutations and CHARGE syndrome: the clinical implications of an expanding phenotype. J. Med. Genet. 48, 334-342. doi: 10.1136/jmg.2010.087106

Blustajn, J., Kirsch, C. F., Panigrahy, A., and Netchine, I. (2008). Olfactory anomalies in CHARGE syndrome: imaging findings of a potential major diagnostic criterion. AJNR Am. J. Neuroradiol. 29, 1266-1269. doi: 10.3174/ajnr. A1099

Bosman, E. A., Penn, A. C., Ambrose, J. C., Kettleborough, R., Stemple, D. L., and Steel, K. P. (2005). Multiple mutations in mouse Chd7 provide models for CHARGE syndrome. Hum. Mol. Genet. 14, 3463-3476. doi: 10.1093/hmg/ ddi375

Bouazoune, K., and Kingston, R. E. (2012). Chromatin remodeling by the CHD7 protein is impaired by mutations that cause human developmental disorders. Proc. Natl. Acad. Sci. U.S.A. 109, 19238-19243. doi: 10.1073/pnas.1213825109

Chalouhi, C., Faulcon, P., Le Bihan, C., Hertz-Pannier, L., Bonfils, P., and Abadie, V. (2005). Olfactory evaluation in children: application to the CHARGE syndrome. Pediatrics 116, e81-e88. doi: 10.1542/peds.2004-1970

Clapier, C. R., and Cairns, B. R. (2009). The biology of chromatin remodeling complexes. Annu. Rev. Biochem. 78, 273-304. doi: 10.1146/annurev.biochem. 77.062706.153223

Dykhuizen, E. C., Hargreaves, D. C., Miller, E. L., Cui, K., Korshunov, A., Kool, M., et al. (2013). BAF complexes facilitate decatenation of DNA by topoisomerase IIalpha. Nature 497, 624-627. doi: 10.1038/nature12146

Engelen, E., Akinci, U., Bryne, J. C., Hou, J., Gontan, C., Moen, M., et al. (2011). Sox 2 cooperates with $\mathrm{Chd} 7$ to regulate genes that are mutated in human syndromes. Nat. Genet. 43, 607-611. doi: 10.1038/ng.825

Feng, W., Kawauchi, D., Korkel-Qu, H., Deng, H., Serger, E., Sieber, L., et al. (2017). Chd7 is indispensable for mammalian brain development through activation of a neuronal differentiation programme. Nat. Commun. 8:14758. doi: 10.1038/ ncomms 14758

Feng, W., Khan, M. A., Bellvis, P., Zhu, Z., Bernhardt, O., Herold-Mende, C., et al. (2013). The chromatin remodeler CHD7 regulates adult neurogenesis via activation of SoxC transcription factors. Cell Stem Cell 13, 62-72. doi: 10.1016/ j.stem.2013.05.002

Gage, F. H. (2000). Mammalian neural stem cells. Science 287, 1433-1438. doi: $10.1126 /$ science.287.5457.1433

Habib, N., Li, Y., Heidenreich, M., Swiech, L., Avraham-Davidi, I., Trombetta, J. J., et al. (2016). Div-Seq: single-nucleus RNA-Seq reveals dynamics of rare adult newborn neurons. Science 353, 925-928. doi: 10.1126/science. aad7038

Hale, C. L., Niederriter, A. N., Green, G. E., and Martin, D. M. (2016). Atypical phenotypes associated with pathogenic CHD7 variants and a proposal for broadening CHARGE syndrome clinical diagnostic criteria. Am. J. Med. Genet. A 170A, 344-354. doi: 10.1002/ajmg.a.37435
Hall, B. D. (1979). Choanal atresia and associated multiple anomalies. J. Pediatr. 95, 395-398. doi: 10.1016/S0022-3476(79)80513-2

He, D., Marie, C., Zhao, C., Kim, B., Wang, J., Deng, Y., et al. (2016). Chd7 cooperates with Sox10 and regulates the onset of CNS myelination and remyelination. Nat. Neurosci. 19, 678-689. doi: 10.1038/nn.4258

Hittner, H. M., Hirsch, N. J., Kreh, G. M., and Rudolph, A. J. (1979). Colobomatous microphthalmia, heart disease, hearing loss, and mental retardation-a syndrome. J. Pediatr. Ophthalmol. Strabismus 16, 122-128.

Hnisz, D., Abraham, B. J., Lee, T. I., Lau, A., Saint-Andre, V., Sigova, A. A., et al. (2013). Super-enhancers in the control of cell identity and disease. Cell 155, 934-947. doi: 10.1016/j.cell.2013.09.053

Ho, L., and Crabtree, G. R. (2010). Chromatin remodelling during development. Nature 463, 474-484. doi: 10.1038/nature08911

Hockemeyer, D., and Jaenisch, R. (2016). Induced pluripotent stem cells meet genome editing. Cell Stem Cell 18, 573-586.

Hurd, E. A., Capers, P. L., Blauwkamp, M. N., Adams, M. E., Raphael, Y., Poucher, H. K., et al. (2007). Loss of Chd7 function in gene-trapped reporter mice is embryonic lethal and associated with severe defects in multiple developing tissues. Mamm. Genome 18, 94-104. doi: 10.1007/s00335-006-0107-6

Hurd, E. A., Poucher, H. K., Cheng, K., Raphael, Y., and Martin, D. M. (2010). The ATP-dependent chromatin remodeling enzyme $\mathrm{CHD} 7$ regulates proneural gene expression and neurogenesis in the inner ear. Development 137, 3139-3150. doi: 10.1242/dev.047894

Husain, A., Begum, N. A., Taniguchi, T., Taniguchi, H., Kobayashi, M., and Honjo, T. (2016). Chromatin remodeller SMARCA4 recruits topoisomerase 1 and suppresses transcription-associated genomic instability. Nat. Commun. 7:10549. doi: 10.1038/ncomms10549

Jiang, X., Zhou, Y., Xian, L., Chen, W., Wu, H., and Gao, X. (2012). The mutation in Chd7 causes misexpression of Bmp4 and developmental defects in telencephalic midline. Am. J. Pathol. 181, 626-641. doi: 10.1016/j.ajpath.2012.05.006

Johansson, M., Rastam, M., Billstedt, E., Danielsson, S., Stromland, K., Miller, M., et al. (2006). Autism spectrum disorders and underlying brain pathology in CHARGE association. Dev. Med. Child Neurol. 48, 40-50. doi: 10.1017/ S0012162206000090

Jones, K. M., Saric, N., Russell, J. P., Andoniadou, C. L., Scambler, P. J., and Basson, M. A. (2015). CHD7 maintains neural stem cell quiescence and prevents premature stem cell depletion in the adult hippocampus. Stem Cells 33, 196-210. doi: 10.1002/stem.1822

Kim, H. G., Kurth, I., Lan, F., Meliciani, I., Wenzel, W., Eom, S. H., et al. (2008). Mutations in CHD7, encoding a chromatin-remodeling protein, cause idiopathic hypogonadotropic hypogonadism and Kallmann syndrome. Am. J. Hum. Genet. 83, 511-519. doi: 10.1016/j.ajhg.2008.09.005

King, I. F., Yandava, C. N., Mabb, A. M., Hsiao, J. S., Huang, H. S., Pearson, B. L., et al. (2013). Topoisomerases facilitate transcription of long genes linked to autism. Nature 501, 58-62. doi: 10.1038/nature12504

Lancaster, M. A., Renner, M., Martin, C. A., Wenzel, D., Bicknell, L. S., Hurles, M. E., et al. (2013). Cerebral organoids model human brain development and microcephaly. Nature 501, 373-379. doi: 10.1038/nature12517

Laurette, P., Strub, T., Koludrovic, D., Keime, C., Le Gras, S., Seberg, H., et al. (2015). Transcription factor MITF and remodeller BRG1 define chromatin organisation at regulatory elements in melanoma cells. Elife 4:e06857. doi: 10.7554/eLife.06857

Layman, W. S., Hurd, E. A., and Martin, D. M. (2010). Chromodomain proteins in development: lessons from CHARGE syndrome. Clin. Genet. 78, 11-20. doi: 10.1111/j.1399-0004.2010.01446.x

Layman, W. S., Hurd, E. A., and Martin, D. M. (2011). Reproductive dysfunction and decreased GnRH neurogenesis in a mouse model of CHARGE syndrome. Hum. Mol. Genet. 20, 3138-3150. doi: 10.1093/hmg/ddr216

Layman, W. S., McEwen, D. P., Beyer, L. A., Lalani, S. R., Fernbach, S. D., Oh, E., et al. (2009). Defects in neural stem cell proliferation and olfaction in Chd7 deficient mice indicate a mechanism for hyposmia in human CHARGE syndrome. Hum. Mol. Genet. 18, 1909-1923. doi: 10.1093/hmg/ddp112

Legendre, M., Gonzales, M., Goudefroye, G., Bilan, F., Parisot, P., Perez, M. J., et al. (2012). Antenatal spectrum of CHARGE syndrome in 40 fetuses with CHD7 mutations. J. Med. Genet. 49, 698-707. doi: 10.1136/jmedgenet-2012-100926

Lin, A. E., Siebert, J. R., and Graham, J. M. Jr. (1990). Central nervous system malformations in the CHARGE association. Am. J. Med. Genet. 37, 304-310. doi: 10.1002/ajmg.1320370303 
Lusser, A., Urwin, D. L., and Kadonaga, J. T. (2005). Distinct activities of CHD1 and ACF in ATP-dependent chromatin assembly. Nat. Struct. Mol. Biol. 12, 160-166. doi: $10.1038 / \mathrm{nsmb} 884$

Lyu, Y. L., and Wang, J. C. (2003). Aberrant lamination in the cerebral cortex of mouse embryos lacking DNA topoisomerase IIbeta. Proc. Natl. Acad. Sci. U.S.A. 100, 7123-7128. doi: 10.1073/pnas.1232376100

Marcos, S., Sarfati, J., Leroy, C., Fouveaut, C., Parent, P., Metz, C., et al. (2014). The prevalence of $\mathrm{CHD} 7$ missense versus truncating mutations is higher in patients with Kallmann syndrome than in typical CHARGE patients. J. Clin. Endocrinol. Metab. 99, E2138-E2143. doi: 10.1210/jc.2014-2110

Martynoga, B., Mateo, J. L., Zhou, B., Andersen, J., Achimastou, A., Urban, N., et al. (2013). Epigenomic enhancer annotation reveals a key role for NFIX in neural stem cell quiescence. Genes Dev. 27, 1769-1786. doi: 10.1101/gad.216804.113

Melicharek, D. J., Ramirez, L. C., Singh, S., Thompson, R., and Marenda, D. R. (2010). Kismet/CHD7 regulates axon morphology, memory and locomotion in a Drosophila model of CHARGE syndrome. Hum. Mol. Genet. 19, 4253-4264. doi: 10.1093/hmg/ddq348

Micucci, J. A., Layman, W. S., Hurd, E. A., Sperry, E. D., Frank, S. F., Durham, M. A., et al. (2014). CHD7 and retinoic acid signaling cooperate to regulate neural stem cell and inner ear development in mouse models of CHARGE syndrome. Hum. Mol. Genet. 23, 434-448. doi: 10.1093/hmg/ddt435

Miller, E. L., Hargreaves, D. C., Kadoch, C., Chang, C. Y., Calarco, J. P., Hodges, C., et al. (2017). TOP2 synergizes with BAF chromatin remodeling for both resolution and formation of facultative heterochromatin. Nat. Struct. Mol. Biol. 24, 344-352. doi: 10.1038/nsmb.3384

Moreno, N., Schmidt, C., Ahlfeld, J., Poschl, J., Dittmar, S., Pfister, S. M., et al. (2014). Loss of Smarc proteins impairs cerebellar development. J. Neurosci. 34, 13486-13491. doi: 10.1523/JNEUROSCI.2560-14.2014

Morris, S. A., Baek, S., Sung, M. H., John, S., Wiench, M., Johnson, T. A., et al. (2014). Overlapping chromatin-remodeling systems collaborate genome wide at dynamic chromatin transitions. Nat. Struct. Mol. Biol. 21, 73-81. doi: 10.1038/ nsmb. 2718

Ninkovic, J., Steiner-Mezzadri, A., Jawerka, M., Akinci, U., Masserdotti, G., Petricca, S., et al. (2013). The BAF complex interacts with Pax6 in adult neural progenitors to establish a neurogenic cross-regulatory transcriptional network. Cell Stem Cell 13, 403-418. doi: 10.1016/j.stem.2013.07.002

Pagon, R. A., Graham, J. M. Jr., Zonana, J., and Yong, S. L. (1981). Coloboma, congenital heart disease, and choanal atresia with multiple anomalies: CHARGE association. J. Pediatr. 99, 223-227. doi: 10.1016/S0022-3476(81)80454-4

Patten, S. A., Jacobs-McDaniels, N. L., Zaouter, C., Drapeau, P., Albertson, R. C., and Moldovan, F. (2012). Role of Chd7 in zebrafish: a model for CHARGE syndrome. PLOS ONE 7:e31650. doi: 10.1371/journal.pone.0031650

Pinto, G., Abadie, V., Mesnage, R., Blustajn, J., Cabrol, S., Amiel, J., et al. (2005). CHARGE syndrome includes hypogonadotropic hypogonadism and abnormal olfactory bulb development. J. Clin. Endocrinol. Metab. 90, 5621-5626. doi: $10.1210 /$ jc.2004-2474

Ram, O., Goren, A., Amit, I., Shoresh, N., Yosef, N., Ernst, J., et al. (2011). Combinatorial patterning of chromatin regulators uncovered by genome-wide location analysis in human cells. Cell 147, 1628-1639. doi: 10.1016/j.cell.2011. 09.057

Sanlaville, D., Etchevers, H. C., Gonzales, M., Martinovic, J., Clement-Ziza, M., Delezoide, A. L., et al. (2006). Phenotypic spectrum of CHARGE syndrome in fetuses with $\mathrm{CHD} 7$ truncating mutations correlates with expression during human development. J. Med. Genet. 43, 211-217. doi: 10.1136/jmg.2005.036160
Schnetz, M. P., Bartels, C. F., Shastri, K., Balasubramanian, D., Zentner, G. E., Balaji, R., et al. (2009). Genomic distribution of CHD7 on chromatin tracks H3K4 methylation patterns. Genome Res. 19, 590-601. doi: 10.1101/gr.08 6983.108

Schnetz, M. P., Handoko, L., Akhtar-Zaidi, B., Bartels, C. F., Pereira, C. F., Fisher, A. G., et al. (2010). CHD7 targets active gene enhancer elements to modulate ES cell-specific gene expression. PLOS Genet. 6:e1001023. doi: 10.1371/journal. pgen.1001023

Sundaramoorthy, R., Hughes, A. L., Singh, V., Wiechens, N., Ryan, D. P., ElMkami, H., et al. (2017). Structural reorganization of the chromatin remodeling enzyme Chd1 upon engagement with nucleosomes. Elife 6:e22510. doi: 10.7554/ eLife. 22510

Tellier, A. L., Cormier-Daire, V., Abadie, V., Amiel, J., Sigaudy, S., Bonnet, D., et al. (1998). CHARGE syndrome: report of 47 cases and review. Am. J. Med. Genet. 76, 402-409. doi: 10.1002/(SICI)1096-8628(19980413)76:5<402::AIDAJMG7>3.0.CO;2-O

Tran, H. G., Steger, D. J., Iyer, V. R., and Johnson, A. D. (2000). The chromo domain protein chdlp from budding yeast is an ATP-dependent chromatinmodifying factor. EMBO J. 19, 2323-2331. doi: 10.1093/emboj/19.10.2323

Van Nostrand, J. L., Brady, C. A., Jung, H., Fuentes, D. R., Kozak, M. M., Johnson, T. M., et al. (2014). Inappropriate p53 activation during development induces features of CHARGE syndrome. Nature 514, 228-232. doi: 10.1038/ nature 13585

Vissers, L. E., van Ravenswaaij, C. M., Admiraal, R., Hurst, J. A., de Vries, B. B., Janssen, I. M., et al. (2004). Mutations in a new member of the chromodomain gene family cause CHARGE syndrome. Nat. Genet. 36, 955-957. doi: 10.1038/ ng1407

Whittaker, D. E., Riegman, K. L., Kasah, S., Mohan, C., Yu, T., Sala, B. P., et al. (2017). The chromatin remodeling factor CHD7 controls cerebellar development by regulating reelin expression. J. Clin. Invest. 127, 874-887. doi: 10.1172/JCI83408

Yu, T., Meiners, L. C., Danielsen, K., Wong, M. T., Bowler, T., Reinberg, D., et al. (2013). Deregulated FGF and homeotic gene expression underlies cerebellar vermis hypoplasia in CHARGE syndrome. Elife 2:e01305. doi: 10.7554/eLife. 01305

Zentner, G. E., Hurd, E. A., Schnetz, M. P., Handoko, L., Wang, C., Wang, Z., et al. (2010). CHD7 functions in the nucleolus as a positive regulator of ribosomal RNA biogenesis. Hum. Mol. Genet. 19, 3491-3501. doi: 10.1093/hmg/ ddq265

Zhang, Y., LeRoy, G., Seelig, H. P., Lane, W. S., and Reinberg, D. (1998). The dermatomyositis-specific autoantigen $\mathrm{Mi} 2$ is a component of a complex containing histone deacetylase and nucleosome remodeling activities. Cell 95, 279-289.

Conflict of Interest Statement: The authors declare that the research was conducted in the absence of any commercial or financial relationships that could be construed as a potential conflict of interest.

Copyright (c) 2017 Feng, Shao and Liu. This is an open-access article distributed under the terms of the Creative Commons Attribution License (CC BY). The use, distribution or reproduction in other forums is permitted, provided the original author(s) or licensor are credited and that the original publication in this journal is cited, in accordance with accepted academic practice. No use, distribution or reproduction is permitted which does not comply with these terms. 